\title{
Continuous Self-Energy of Ions at the Dielectric Interface
}

\author{
Rui Wang and Zhen-Gang Wang* \\ Division of Chemistry and Chemical Engineering, California Institute of Technology, Pasadena, California 91125, USA
}

(Received 20 October 2013; published 2 April 2014)

\begin{abstract}
By treating both the short-range (solvation) and long-range (image force) electrostatic forces as well as charge polarization induced by these forces in a consistent manner, we obtain a simple theory for the selfenergy of an ion that is continuous across the interface. Along with nonelectrostatic contributions, our theory enables a unified description of ions on both sides of the interface. Using intrinsic parameters of the ions, we predict the specific ion effect on the interfacial affinity of halogen anions at the water-air interface, and the strong adsorption of hydrophobic ions at the water-oil interface, in agreement with experiments and atomistic simulations.
\end{abstract}

DOI: 10.1103/PhysRevLett.112.136101

PACS numbers: 82.45.Gj, 05.20.-y, 61.20.Qg, 68.03.Cd

The interfacial activities of salt ions are of great importance in physical chemistry, colloidal science, and biophysics [1]. Many interfacial phenomena, such as the surface tension of the electrolyte solution [2], salt effects on bubble coalescence [3], and the effectiveness of salts on the stability of protein solutions and colloidal suspensions [4], exhibit strong dependence on the chemical identity of the ions. Although this "specific ion effect" has been known for over a century [5], a systematic, unified, and predictive theory remains an outstanding challenge. Current theories are system dependent and require adjustable parameters to force-fit experimental data [6-10].

A key factor that determines the ion distribution at the dielectric interface and other interfacial properties is the self-energy of a single ion [11]. The self-energy consists of electrostatic and nonelectrostatic contributions, such as cavity energy, hydration, and dispersion forces. While the effects and the theoretical treatments of these nonelectrostatic contributions are still debatable [6], the constituent components in the electrostatic self-energy have become clear in recent years [12-14]. The problem is then in the accurate and consistent treatment of the electrostatic effects. Such a treatment is essential both because the electrostatic part is a major component in the self-energy of an ion, and because the relative importance of the nonelectrostatic contributions can only be evaluated when the electrostatic contribution is treated accurately.

A major contribution in the electrostatic self energy is the image interaction, whose treatment was pioneered by Wagner, Onsager, and Samaras (WOS) [15]. The WOS theory predicts depletion of ions from the water-air interface due to the image charge repulsion and qualitatively explains the increase of surface tension with the salt concentration. However, this theory fails to capture the initial decrease with salt concentration in the surface tension known as the Jones-Ray effect [16], and the systematic dependence on the identity of the ions [2]. A major weakness in the WOS theory and its subsequent modifications is modeling the ion as a point charge, which results in a discontinuous self-energy across the dielectric interface. The self-energy diverges to positive infinity on approaching the interface from the water side and to negative infinity on approaching from the air(oil) side. To avoid this unrealistic behavior, the ion distribution is artificially restricted to lie only in the water phase, which makes the theory inapplicable to hydrophobic ions and liquid-liquid interfaces. This artificial cutoff also affects the electrostatic potential gradient across the interface, which is shown essential to the Jones-Ray effect [17].

Another important effect is the finite polarizability of the ions. Simulation by Jungwirth and Tobias [18] showed that the polarizability of ions is a key contribution to their differential affinity to the interface. Levin and co-workers [12-14] developed a model of polarizable ions near a dielectric interface that are able to explain several interfacial properties of aqueous electrolyte solutions. In their model, charge polarization in the ion is included to optimize the short-range Born energy. However, near a dielectric interface, the long-range image force can be sufficiently strong to contribute to charge polarization. Furthermore, their model does not account for the image force on the air(oil) side of the interface, thus making it difficult to extend the theory to hydrophobic ions and liquid-liquid interfaces.

In this Letter, we present a unified theory that treats all the electrostatic contributions: the Born solvation energy, the image charge interaction, and ion polarizability in a single, consistent framework. Along with the relevant nonelectrostatic contributions, we apply our theory to air-water and liquid-liquid interfaces.

Electrostatic self-energy.-We consider a single ion in the vicinity of a sharp interface, located at $z=0$, between two semi-infinite regions $\left(\Re_{1}\right.$ and $\left.\Re_{2}\right)$ with respective dielectric constant $\varepsilon_{1}$ and $\varepsilon_{2}\left(\varepsilon_{1}>\varepsilon_{2}\right)$. We take the elementary charge $e$ as the unit of charge, and kT as the unit of energy. The ion is taken as a sphere of radius $a$ 
centered at $\mathbf{r}_{c}$, with charge distribution $\rho\left(\mathbf{r}, \mathbf{r}_{c}\right)$, which satisfies $\int d \mathbf{r} \rho\left(\mathbf{r}, \mathbf{r}_{c}\right)=\nu_{ \pm}$with $\nu_{ \pm}$the valency of the ion ( + for the cation and - for the anion). The ion is polarizable; therefore, the charge distribution will be self-adjusted to the local dielectric environment.

The electrostatic self-energy $u_{\mathrm{el}}$ can be written as two parts: $u_{\mathrm{el}}=u_{\text {int }}+u_{\mathrm{pol}}$, where $u_{\text {int }}$ accounts for the sum of the Coulomb interactions in the constituent charges on the ion and $u_{\mathrm{pol}}$ is the energy cost of charge polarization. $u_{\mathrm{int}}$ is given by

$$
u_{\mathrm{int}}\left(\mathbf{r}_{c}\right)=2 \pi l_{B} \int d \mathbf{r} \int d \mathbf{r}^{\prime} \rho\left(\mathbf{r}, \mathbf{r}_{c}\right) G\left(\mathbf{r}, \mathbf{r}^{\prime}\right) \rho\left(\mathbf{r}^{\prime}, \mathbf{r}_{c}\right),
$$

where $l_{B}=e^{2} / 4 \pi \varepsilon_{0} \mathrm{kT}$ is the Bjerrum length in the vacuum and $\varepsilon_{0}$ is the vacuum permitivity. $G\left(\mathbf{r}, \mathbf{r}^{\prime}\right)$ is the Green's function: the electrostatic potential at $\mathbf{r}$ due to a unit point charge at $\mathbf{r}^{\prime}$. It satisfies the Poisson equation $-\nabla$. $\left[\varepsilon(\mathbf{r}) \nabla G\left(\mathbf{r}, \mathbf{r}^{\prime}\right)\right]=\delta\left(\mathbf{r}-\mathbf{r}^{\prime}\right)$. Depending on whether $\mathbf{r}$ and $\mathbf{r}^{\prime}$ are in the same region, $G\left(\mathbf{r}, \mathbf{r}^{\prime}\right)$ is given by

$$
G\left(\mathbf{r}, \mathbf{r}^{\prime}\right)= \begin{cases}\frac{1}{4 \pi \varepsilon_{\alpha}\left|\mathbf{r}-\mathbf{r}^{\prime}\right|}+\frac{\Delta_{\alpha \beta}}{4 \pi \varepsilon_{\alpha}\left|\mathbf{r}-\mathbf{r}^{\star}\right|} & \mathbf{r}, \mathbf{r}^{\prime} \in \Re_{\alpha} \\ \frac{1}{2 \pi\left(\varepsilon_{\alpha}+\varepsilon_{\beta}\right)\left|\mathbf{r}-\mathbf{r}^{\prime}\right|} & \mathbf{r} \in \Re_{\beta}, \mathbf{r}^{\prime} \in \Re_{\alpha},\end{cases}
$$

$\alpha$ and $\beta$ can be either 1 or 2 , and $\Delta_{\alpha \beta}=\left(\varepsilon_{\alpha}-\varepsilon_{\beta}\right) /\left(\varepsilon_{\alpha}+\varepsilon_{\beta}\right)$ is the dielectric contrast. $\mathbf{r}^{\star}=\left(x^{\prime}, y^{\prime},-z^{\prime}\right)$ is the location of the image of $\mathbf{r}^{\prime}$ with respect to the interface. The first term on the right-hand side of Eq. (2) is the direct Coulomb interaction and will generate the local Born solvation energy upon integration over the charge distribution. The last term in the first line of Eq. (2) is the image charge interaction, which can be either positive or negative depending on whether the point charge is located on the high dielectric side or low dielectric side; thus, it either enhances or counteracts the solvation energy effect.

In the point-charge model $\rho\left(\mathbf{r}, \mathbf{r}_{c}\right)=\nu_{ \pm} \delta\left(\mathbf{r}-\mathbf{r}_{c}\right)$, Eq. (1) gives $u_{\text {int }}\left(\mathbf{r}_{c}\right)=2 \pi l_{B} \nu_{ \pm}^{2} G\left(\mathbf{r}_{c}, \mathbf{r}_{c}\right)$, which produces divergences in both the local Born solvation energy and in the image charge interaction as $z_{c} \rightarrow 0$ from either side of the interface. The use of a finite charge distribution avoids both types of divergences. Figure 1 shows the result for the electrostatic self-energy, $u_{\mathrm{el}}^{(0)}$, calculated for a nonpolarizable, uniform surface charge distribution on the ion (thus $u_{\mathrm{el}}^{(0)}=u_{\mathrm{int}}$ ). For comparison, we include the results from the point-charge model, adjusted by the bulk Born energy $\nu_{ \pm}^{2} l_{B} / 2 a \varepsilon_{\alpha}$ on each side. While $u_{\mathrm{el}}^{(0)}$ calculated by the two models are consistent in the bulk region $\left(\left|z_{c}\right|>a\right)$, qualitative and dramatic differences are seen in the interfacial region - the most relevant region for the interfacial activities of the ions. Interestingly, $u_{\mathrm{el}}^{(0)}$ for an ion located exactly at the interface $\left(z_{c}=0\right)$ is significantly lower than the mean of the Born energy in two bulk regions, reflecting the

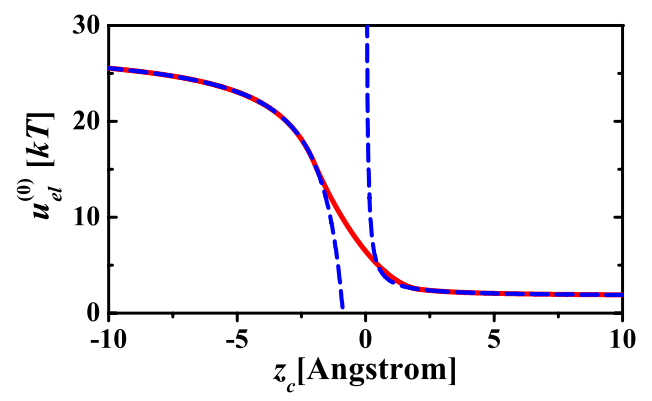

FIG. 1 (color online). Electrostatic self-energy, $u_{\mathrm{el}}^{(0)}$, of a monovalent ion with uniform surface charge distribution, calculated by our model (solid line) and the point charge model (dashed line). $\varepsilon_{1}=80, \varepsilon_{2}=5$, and $a=2 \AA$.

asymmetry in the image force between the two dielectric media.

Polarization of the ion allows the charge distribution to self-adjust to its local dielectric environment, which decreases $u_{\text {int }}$ relative to that for a fixed uniform charge distribution. However, this redistribution incurs an energy penalty $u_{\mathrm{pol}}$. Levin [12] proposed a phenomenological model for $u_{\mathrm{pol}}$ by taking reference to the perfectly conducting sphere and making a Landau type of symmetry argument to describe this energy penalty. In our notation $u_{\mathrm{pol}}$ is

$$
u_{\mathrm{pol}}\left(\mathbf{r}_{c}\right)=\frac{\left(\gamma_{0}-\gamma\right)}{2 v \gamma} \int d \mathbf{r}\left[\frac{\rho\left(\mathbf{r}, \mathbf{r}_{c}\right)}{\rho_{0}}-1\right]^{2}
$$

where $\gamma$ is the polarizability of the ion, $\gamma_{0}\left(=a^{3}\right)$ is the polarizability of a perfectly conducting sphere of the same radius as the ion [19], $v$ is the volume of the ion, and $\rho_{0}$ is the charge density for the uniform spherical distribution on the ion surface. The form of the coefficient in Eq. (3) was so constructed as to reproduce the known limiting behavior, i.e., that it should be zero for the perfectly conducting sphere and infinity for a nonpolarizable ion.

Putting together Eqs. (1) and (3), we obtain the general expression for $u_{\mathrm{el}}$ with arbitrary charge distribution on the ion. The optimal distribution is then obtained from $\delta u_{\mathrm{el}}\left(\mathbf{r}_{c}\right) / \delta \rho\left(\mathbf{r}, \mathbf{r}_{c}\right)=0$. To avoid the complexity of solving the high dimensional integral equation from this condition, we make a variational trial function for $\rho\left(\mathbf{r}, \mathbf{r}_{c}\right)$. We assume that polarization apportions, respectively, $f$ and $1-f$ of the total ionic charge $(f \in[0,1])$ uniformly to the two hemispheres of the ion separated by the $x y$ plane at $z_{c}$, i.e.,

$$
\rho\left(\mathbf{r}, \mathbf{r}_{c}\right)= \begin{cases}2 f \rho_{0}(\mathbf{r}) & \text { for } z \geq z_{c} \\ 2(1-f) \rho_{0}(\mathbf{r}) & \text { for } z<z_{c}\end{cases}
$$

where $\rho_{0}(\mathbf{r})=\nu_{ \pm} \delta\left(\left|\mathbf{r}-\mathbf{r}_{c}\right|-a\right) / 4 \pi a^{2}$ is the uniform surface distribution on the sphere. The deviation of $f$ from $1 / 2$ measures the degree of polarization of the ionic charge. Substituting the trial function Eq. (7) into Eqs. (1) and (3), 
$u_{\mathrm{el}}\left(\mathbf{r}_{c}\right)$ can be simplified to a quadratic function of $f$, which can be easily minimized to yield a position-dependent charge fraction $f\left(\mathbf{r}_{c}\right)$. This optimal charge fraction $f\left(\mathbf{r}_{c}\right)$ is then used to evaluate $u_{\mathrm{el}}\left(\mathbf{r}_{c}\right)$. Since the electrostatic interaction includes the local Born solvation energy and the long-range image force, the resulting polarization reflects the combined effects of these terms. Figure 2 shows the charge polarization and the electrostatic selfenergy of $I^{-}\left(a_{I^{-}}=2.26 \AA \gamma_{I^{-}}=6.9 \AA^{3}\right)$. In the immediate vicinity of the interface $\left(\left|z_{c}\right|<a\right), I^{-}$is highly polarized. Charge polarization significantly lowers $u_{\mathrm{el}}$ compared with the nonpolarizable ion. Beyond the immediate vicinity of the interface $\left(\left|z_{c}\right|>a\right)$, polarization is driven by the long-range image force, and $f$ decays to $1 / 2$ as the ion approaches the bulk. While the effect of charge polarization on $u_{\mathrm{el}}$ is small on the high dielectric side beyond $z_{c}=a, u_{\mathrm{el}}$ of the polarizable ion is appreciably lower than the nonpolarizable ion on the low dielectric side slightly beyond $z_{c}=-a$, as a result of stronger and longerrange image force in this region.

For comparison, in Fig. 2(b) we also include results from Levin's polarizable ion model [12]. $u_{\mathrm{el}}$ in Levin's theory only extends to $z_{c}=-a$, whereas our theory yields a continuous $u_{\mathrm{el}}$ across the interface to the bulk air(oil) phase; this will be important when there is appreciable ion partition in the oil phase. In addition, $u_{\mathrm{el}}$ on the low dielectric side is significantly higher in our theory than from Levin's theory because the relocation of charge from $z<0$ to $z>0$ changes the image force from attractive to repulsive, which is not accounted for in Levin's theory. The difference becomes more pronounced with increasing dielectric contrast.

The calculations so far concern only the electrostatic contributions to the self-energy, which will not be affected by the addition of nonelectrostatic effects. The total selfenergy of the ion is the sum of the electrostatic and nonelectrostatic parts:

$$
u\left(\mathbf{r}_{c}\right)=u_{\mathrm{el}}\left(\mathbf{r}_{c}\right)+u_{\mathrm{ne}}\left(\mathbf{r}_{c}\right) .
$$
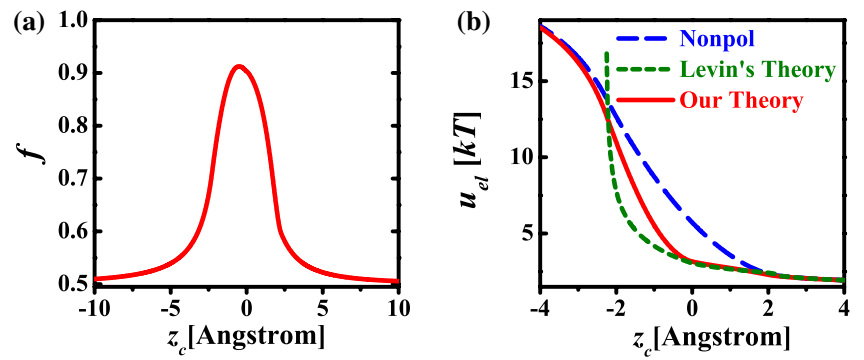

FIG. 2 (color online). (a) Charge polarization $f$, and (b) electrostatic self-energy $u_{\mathrm{el}}$, for $\mathrm{I}^{-}$. For comparison, we include Levin's theory [12] and a nonpolarizable ion of the same radius as $\mathrm{I}^{-}$ (dashed line). $\varepsilon_{1}=80, \varepsilon_{2}=5$.
We now include the appropriate nonelectrostatic contributions to discuss the interfacial behavior of different ions at the water-air and water-oil interfaces, respectively.

Water-air interface.-For the nonelectrostatic selfenergy at the water-air interface, we take the simplest form of cavity energy $[12,20]$, which is the work required to create a cavity for the ion where the water molecules are excluded. It is given by $[12,20]$

$$
u_{\mathrm{ne}}^{w / a}\left(\mathbf{r}_{c}\right)= \begin{cases}\kappa a^{3} & z_{c} \geq a \\ \frac{\kappa a^{3}}{4}\left(\frac{z_{c}}{a}+1\right)^{2}\left(2-\frac{z_{c}}{a}\right) & a>z_{c} \geq-a, \\ 0 & z_{c}<-a\end{cases}
$$

with $\kappa \approx 0.3 \AA^{-3}$ from bulk simulation [21]. $u_{\text {ne }}^{w / a}$ provides the driving force for the ion to migrate from the bulk water to the interface; this driving force is larger for larger ions. The self-energy profile of the ion across the interface is determined by the competition between the cavity energy and the electrostatic self-energy, the former preferring the ion to reside on the air side and the latter favoring it being on the aqueous side.

Figure 3(a) shows $u$ for four halogen anions and the alkali-metal $\mathrm{Na}^{+}$. We use the Born radius 2.26, 2.05, 1.91, 1.46 , and $1.80 \AA[22]$, and the polarizability $6.90,4.53$, $3.50,0.97$, and $0.18 \AA^{3}[18]$, respectively, for $\mathrm{I}^{-}, \mathrm{Br}^{-}, \mathrm{Cl}^{-}$, $\mathrm{F}^{-}$, and $\mathrm{Na}^{+}$. For the larger and more polarizable ions, such as $\mathrm{I}^{-}$and $\mathrm{Br}^{-}$, the gain in cavity energy at the relatively low cost of electrostatic self-energy leads to a local minimum at the interface in the self-energy profile, which is consistent with the result of MD simulation using a polarizable potential model [23]. For small and less polarizable ions, such as $\mathrm{F}^{-}$and $\mathrm{Na}^{+}, u$ is monotonic and increases rapidly as the ion moves from the aqueous phase to air. Our theory predicts a more repulsive $u_{\mathrm{el}}$ for these ions on the water side than in Ref. [12], which has the same qualitative effect as the hydration effect considered in Refs. $[13,14]$ for predicting the surface tension. For more complex ions such as $\mathrm{IO}_{3}^{-}$, explicit treatment of hydration may be necessary $[13,14]$. However, the quantitative importance of hydration needs to be reevaluated with our more accurate electrostatic self-energy.
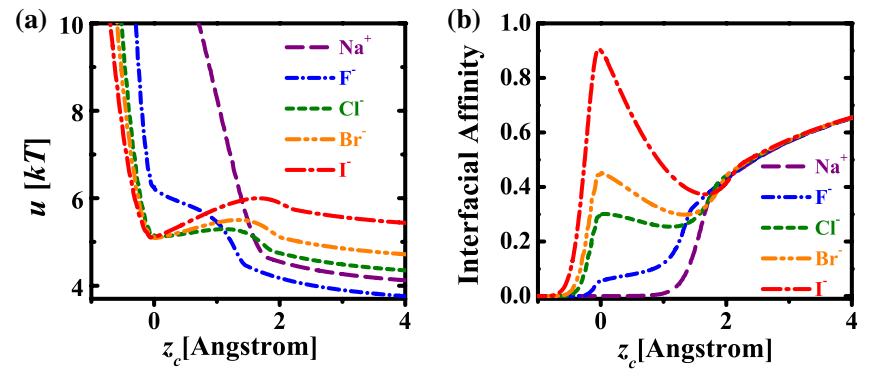

FIG. 3 (color online). (a) Self-energy and (b) interfacial affinity of $\mathrm{F}^{-}, \mathrm{Cl}^{-}, \mathrm{Br}^{-}, \mathrm{I}^{-}$, and $\mathrm{Na}^{+}$at the water-air interface. $\varepsilon_{1}=80, \varepsilon_{2}=1$. 
The self-energy of an ion is closely related to the concentration profile of the ions. While a full treatment has to include the interaction between the ions, which leads to screening of the image forces, we can obtain a qualitative picture of the ion distribution by defining the interfacial affinity as $e^{-\left[u\left(z_{c}\right)-u(\infty)\right]}$ to characterize the relative probability of finding the ion in the interfacial region to the bulk. In Fig. 3(b), we show the interfacial affinity for the halogen anions and $\mathrm{Na}^{+}$. It is clear that our theory captures the known specific ion effect, which follows precisely the reverse Hofmeister series: $\mathrm{I}^{-}>\mathrm{Br}^{-}>\mathrm{Cl}^{-}>\mathrm{F}^{-}[2,5]$. The local peak in the interfacial affinity of $\mathrm{I}^{-}$and $\mathrm{Br}^{-}$ions is consistent with results of electron spectroscopy experiments [24] and computer simulations using polarizable fields [18]. In addition, the interfacial affinity of halogen anions is larger than that of $\mathrm{Na}^{+}$, from which we expect local charge separation and an induced electrical double layer at the interface in a $\mathrm{Na} X$ solution, with the halogen anions accumulating right around the location of the interface and the $\mathrm{Na}^{+}$ions next to it on the water side. The electrostatic potential gradient due to charge separation has been shown to be key to explaining the Jones-Ray effect [17].

Water-oil interface.-With a continuous self-energy, our theory naturally applies to the liquid-liquid interface. In addition to cavity energy, dispersion forces have been suggested to be an important contribution to the nonelectrostatic self-energy at the water-oil interface $[6,7,14]$. These nonelectrostatic contributions set a chemical potential difference between the two bulk phases in addition to the Born energy difference. Phenomenologically, these nonelectrostatic effects can be captured by a single parameter $B$ with a crossover in the interfacial region that can be approximated by the interpolation scheme proposed by Levin and co-workers [12-14]. Similar to Eq. (6), we may write the nonelectrostatic self-energy in the form

$$
u_{\mathrm{ne}}^{w / o}\left(\mathbf{r}_{c}\right)=\left\{\begin{array}{ll}
B & z_{c} \geq a \\
\frac{B}{4}\left(\frac{z_{c}}{a}+1\right)^{2}\left(2-\frac{z_{c}}{a}\right) & a>z_{c} \geq-a . \\
0 & z_{c}<-a
\end{array} .\right.
$$

Restricting our consideration to cavity energy and dispersion force, and taking the reference energy to be 0 in the bulk oil, $B=v^{w}-v^{o}+A_{\text {eff }}\left(\gamma / \gamma_{0}\right)$ [14], where $v^{w}$ is the cavity energy in water, which scales with the cavity volume for small cavity sizes $(a<4 \AA)$ and with the surface area for larger cavities [20]. $v^{o}$ is the cavity energy in oil, which is primarily due to the surface energy between the ion and oil $[25,26] . A_{\text {eff }}$ is the effective Hamaker constant for the water-oil interface, estimated to be about 4 kT [14] for a typical oil-water system. Alternatively, we can treat $B$ as an adjustable parameter from the bulk partitioning of the ions between water and oil.

We defer a more general study of ions at the water-oil interface to a future study. Here we consider a special case of hydrophobic ions. Schlossman and co-workers observed strong adsorption of hydrophobic ions at the water-oil
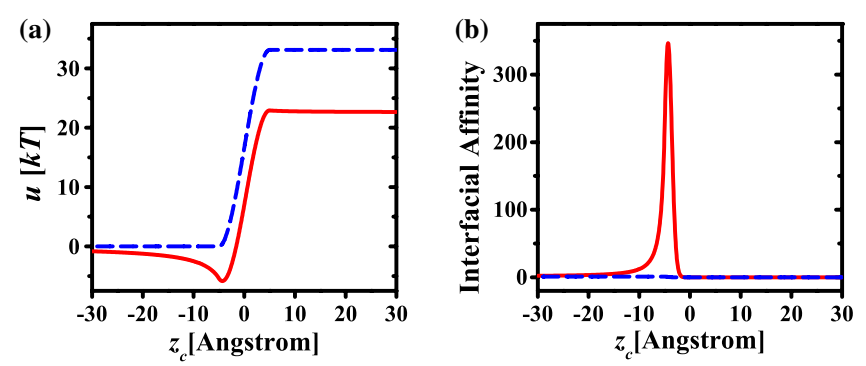

FIG. 4 (color online). (a) Self energy and (b) interfacial affinity of a hydrophobic ion at the water-oil interface relative to the bulk oil. The dashed line shows the results calculated by the nonelectrostatic contribution alone. $a=5 \AA, \gamma / \gamma_{0}=0.5, \varepsilon_{1}=80$, and $\varepsilon_{2}=5$. $B=33 \mathrm{kT}$ [27].

interface by x-ray reflectivity [9], from which it is inferred that there is an attractive well for the self-energy on the oil side. However, no explanation has been given to the origin of this attractive well. Within our theory, this phenomenon can be easily understood as arising from the long-range image charge attraction of the hydrophobic ions in the lowdielectric oil phase. Figure 4 shows the self-energy and interfacial affinity of a hydrophobic ion calculated by our theory with $B=33 \mathrm{kT}$, estimated using $A_{\text {eff }}=4 \mathrm{kT}$, surface tension of water and surface tension of the oil used in the experiment [27]. As the ion approaches the interface from the oil side, $u$ decreases because of the image charge attraction, and then increases rapidly due to the unfavorable contact with the aqueous environment. The peak in the interfacial activity on the oil side of the interface corresponds to minimum in the self-energy with depth of about $5.8 \mathrm{kT}$, in good agreement with the experimental results [9]. We note that although the choice of $B$ will affect bulk partitioning of the ions, the depth of the attractive well is quite insensitive to the precise numerical value as long as $B$ is large enough to ensure hydrophobicity of the ion. This clearly demonstrates the electrostatic origin of the strong adsorption of hydrophobic ions on the oil side of the interface, as the nonelectrostatic contributions (as depicted by the dashed line) do not contain an attractive well.

In conclusion, by treating both the short-range (solvation) and long-range (image force) electrostatic forces as well as charge polarization induced by these forces in a consistent manner, we obtain a simple continuous electrostatic self-energy across the interface, making it applicable to both water-air and liquid-liquid interfaces. A systematic and accurate treatment of the electrostatic self-energy is essential for evaluating the relative importance of the nonelectrostatic contributions. Combining the electrostatic self-energy with existing models for nonelectrostatic contributions, we are able to explain a number of interfacial specific ion effects using the intrinsic parameters of the ion, such as the valency, radius, and polarizability. The selfenergy model developed here provides the essential ingredient in a complete theory to treat ions at finite concentration, via, e.g., the weak coupling theory [28] or 
modified Poisson-Boltzmann theory [29], to describe the phenomena mentioned in the beginning of this Letter.

Acknowledgment is made to the donors of the American Chemical Society Petroleum Research Fund for partial support of this research. We thank Professor Yan Levin for many helpful discussions.

*zgw@ caltech.edu

[1] J. N. Israelachvili, Intermolecular and Surface Forces (Academic Press, New York, 1992).

[2] W. Kunz, P. Lo Nostro, and B. W. Ninham, Curr. Opin. Colloid Interface Sci. 9, 1 (2004); B. C. Garrett, Science 303, 1146 (2004).

[3] V. S. J. Craig, B. W. Ninham, and R. M. Pashley, Nature (London) 364, 317 (1993).

[4] F. W. Tavares, D. Bratko, and J. M. Prausnitz, Curr. Opin. Colloid Interface Sci. 9, 81 (2004); M. Gradzielski, Curr. Opin. Colloid Interface Sci. 9, 256 (2004).

[5] F. Hofmeister, Arch. Exp. Pathol. Pharmakol. 24, 247 (1888).

[6] P. L. Nostro and B. W. Ninham, Chem. Rev. 112, 2286 (2012).

[7] M. Boström, D. R. M. Williams, and B. W. Ninham, Langmuir 17, 4475 (2001).

[8] M. Manciu and E. Ruckenstein, Adv. Colloid Interface Sci. 105, 63 (2003).

[9] N. Laanait, M. Mihaylov, B. Hou, H. Yu, P. Vanysek, M. Meron, B. Lin, I. Benjamin, and M. L. Schlossman, Proc. Natl. Acad. Sci. U.S.A. 109, 20326 (2012); N. Laanait, J. Yoon, B. Hou, P. Vanysek, M. Meron, B. Lin, G. Luo, I. Benjamin, and M. L. Schlossman, J. Chem. Phys. 132, 171101 (2010); G. Luo, S. Malkova, J. Yoon, D. G. Schultz, B. Lin, M. Meron, I. Benjamin, P. Vanýsek, M. L. Schlossman, Science 311, 216 (2006); B. Hou, N. Laanait, H. Yu, W. Bu, J. Yoon, B. Lin, M. Meron, G. Luo, P. Vanysek, and M. L. Schlossman, J. Phys. Chem. B 117, 5365 (2013).

[10] M. Bier, J. Zwanikken, and R.van Roij, Phys. Rev. Lett. 101, 046104 (2008); D. S. Dean and R. R. Horgan, Phys. Rev. E 69, 061603 (2004)

[11] Z. -G. Wang, Phys. Rev. E 81, 021501 (2010).

[12] Y. Levin, Phys. Rev. Lett. 102, 147803 (2009).

[13] Y. Levin, A. P. dos Santos, and A. Diehl, Phys. Rev. Lett. 103, 257802 (2009); Langmuir 26, 10778 (2010).
[14] A. P. dos Santos and Y. Levin, Langmuir 28, 1304 (2012); Faraday Discuss. 160, 75 (2013).

[15] C. Wagner, Phys. Z. 25, 474 (1924); L. Onsager and N. N. T. Samaras, J. Chem. Phys. 2, 528 (1934).

[16] G. Jones and W. A. Ray, J. Am. Chem. Soc. 59, 187 (1937); P. B. Petersen and R. J. Saykally, J. Am. Chem. Soc. 127, 15446 (2005).

[17] A. Onuki, J. Chem. Phys. 128, 224704 (2008); R. Wang and Z.-G. Wang, J. Chem. Phys. 135, 014707 (2011).

[18] P. Jungwirth and D. J. Tobias, J. Phys. Chem. B 106, 6361 (2002); Chem. Rev. 106, 1259 (2006).

[19] J. D. Jackson, Classical Electrodynamics (Wiley, New York, 1999).

[20] K. Lum, D. Chandler, and J. D. Weeks, J. Phys. Chem. B 103, 4570 (1999); D. Chandler, Nature (London) 437, 640 (2005).

[21] S. Rajamani, T. M. Truskett, and S. Garde, Proc. Natl. Acad. Sci. U.S.A. 102, 9475 (2005).

[22] W. M. Latimer, K. S. Pitzer, and C. M. Slansky, J. Chem. Phys. 7, 108 (1939).

[23] L. X. Dang and T.-M. Chang, J. Phys. Chem. B 106, 235 (2002); T.-M. Chang and L.X. Dang, Chem. Rev. 106, 1305 (2006).

[24] S. Ghosal, J.C. Hemminger, H. Bluhm, B. S. Mun, E. L. D. Hebenstreit, G. Ketteler, D. F. Ogletree, F. G. Requejo, M. Salmeron, Science 307, 563 (2005).

[25] R. A. Pierotti, Chem. Rev. 76, 717 (1976).

[26] T. T. Duignan, D. F. Parsons, and B. W. Ninham, J. Phys. Chem. B 117, 9421 (2013).

[27] For this large ion, the cavity energy is proportional to its surface area [20]: $v^{w}-v^{o}=4 \pi a^{2}\left(\sigma^{i w}-\sigma^{i o}\right)$, where $\sigma^{i w}$ and $\sigma^{i o}$ are, respectively, the ion-water and ion-oil interfacial energy. We estimate $\sigma^{i w}$ and $\sigma^{i o}$ in two ways. Taking the ion as a cavity (vacuum), these two surface energies can be approximated by the surface tension of water $\sigma^{w}=$ $72.8 \mathrm{mN} / \mathrm{m}$ and the oil (1,2-dichloroethane [9]) $\sigma^{o}=38.75 \mathrm{mN} / \mathrm{m}$. This yields $B \approx 33 \mathrm{kT}$. If we take the hydrophobic ion to be of the same material as the oil, then $\sigma^{i o}=0 . \sigma^{i w}=\sigma^{o w}$, where $\sigma^{o w}=28.2 \mathrm{mN} / \mathrm{m}$ is the interfacial tension between water and 1,2-Dichloroethane [9]. This estimate gives $B \approx 28 \mathrm{kT}$.

[28] S. L. Carnie and G. M. Torrie, Adv. Chem. Phys. 56, 141 (1984).

[29] D. Horinek and R. R. Netz, Phys. Rev. Lett. 99, 226104 (2007). 